\title{
Right-left confusion in the adult: A verbal labeling effect
}

\author{
M. JEANNE SHOLL and HOWARD E. EGETH \\ Johns Hopkins University, Baltimore, Maryland 21218
}

\begin{abstract}
Directional judgments are typically slower when relative location is described by the words "east" and "west" or "right" and "left" than when described by the words "north" and "south" or "up" and "down." A series of experiments are reported that disentangle verbal from perceptual encoding explanations for right-left difficulty. Overall, our results support a verbal encoding explanation for right-left confusion in the adult. Experiments 1-3 demonstrate that in a response-differentiation task, it is response to the labels "north," "east," "south," and "west" that is responsible for right-left confusion. In addition, Experiments 4-6 demonstrate that right-left difficulty in a mirror image discrimination task is contingent on the use of directional labels. (The data also suggest that it may be more difficult to deal with "up," "down," "left," and "right" than with "north," "south," "east," and "west.") The data are interpreted as inconsistent with a bilateral symmetry explanation for right-left confusion.
\end{abstract}

A number of recent studies indicate that when adults judge the relative locations of objects, their speed and accuracy depends upon the axis of judgment. In general, judgments made with respect to the north-south or up-down axis are superior to judgments made with respect to the east-west or right-left axis. The underlying commonality of these studies is that in each, subjects were required to judge the spatial location or orientation of an object given a verbal description of direction. When direction is described by north-south or up-down, response latency is faster than when direction is described by east-west or right-left. This appears to be the case for single-element and multielement perceptual arrays (Farrell, 1979; Maki, Grandy, \& Hauge, 1979; Maki, Maki, \& Marsh, 1977), schematics of the compass rose (Loftus, 1978), and semantic or map memory (Maki, 1979a; Maki et al., 1977).

The difficulty adults experience when making horizontal judgments is most likely a vestige of the profound difficulty young children have in discriminating right from left (e.g., Corballis \& Beale, 1976). It is generally assumed that adults have outgrown any early trouble they may have had in telling right from left (Corballis \& Beale, 1976); however, with sensitive techniques, such as chronometric analysis, the above studies have demonstrated what seems to be the residual effects of right-left confusion.

This research was supported in part by a grant from the Army Research Institute (DSSW-MDW-903-79-G-002) to Howard Egeth. The authors would like to thank Michael Corballis for his helpful comments on an earlier draft of this paper. We also thank Ronald Cammarata, Scott Dresher, and Karmen Johnson for their help in running subjects and analyzing data. Requests for reprints should be sent to Howard Egeth, Department of Psychology, Johns Hopkins University, Baltimore, Maryland 21218.
Research within an information processing framework has focused on locating the cause of right-left confusion in the adult within one of the four processing stages identified in H. Clark and Chase's (1972) stage analysis of a sentence-picture comparison task. Thus far, the advantage of up-down over right-left judgments, which we will subsequently call the right-left effect, has been ascribed to the verbal encoding stage (Maki et al., 1979), the perceptual encoding stage (Farrell, 1979), and the comparison stage of processing (Maki et al., 1979). To our knowledge, no one has proposed that the response-generation stage is the locus of the right-left effect. The present series of experiments isolates and examines the relative contributions of the verbal and perceptual encoding stages to the rightleft effect.

Farrell (1979, Experiment 3), after demonstrating a right-left effect in a simple go/no-go mirror image discrimination task, has argued that his data support a perceptual encoding explanation of the effect. In Farrell's task, each block of trials contained either pairs of up and down or right and left arrows. Within a block, subjects made a buttonpressing response to one arrow, the target arrow, and refrained from responding to the other arrow. Since performance in this task does not require accessing verbal labels, Farrell concluded that the longer reaction times (RTs) to right-left mirror images must be due to a difficulty in perceptually encoding horizontal stimuli.

Theoretically, a perceptual explanation for right-left confusion appeals to symmetries inherent in the environment (H.Clark, 1973) and/or the organism (H. Clark, 1973; Mach, 1886/1959; Noble, 1968). That left-right confusion might arise from the anatomical symmetry of the organism was originally advocated by Mach (1886/1959), who ascribed the child's propensity 
to confuse the letters $p$ and $q$ or $b$ and $d$ to the symmetry of the oculomotor apparatus. More recently, a theory has been developed by Noble (1968) that predicts, on the basis of the symmetry of the nervous system, that right-left mirror images should be more difficult to discriminate than up-down mirror images. Noble's theory is based on anatomical evidence that the commissures connect mirror image points in the two cerebral hemispheres, and it rests on the assumption that the topographic organization of the retina is maintained more centrally than the striate cortex. As a consequence, visual information, input and represented topographically in each hemisphere, is right-left reversed and represented in mirror image form when transferred to the other hemisphere. Hence, a stimulus is perceptually encoded within each hemisphere in both its veridical (i.e., via direct input) and right-left mirror image (i.e., via input from the contralateral hemisphere) forms. Since right-left reversal of a vertical arrow (e.g., $V$ ) will not change its orientation, a bilaterally symmetric organism should have no difficulty discriminating between up-down mirror images. However, right-left reversal of horizontal arrows (e.g., >) renders right-left mirror images perceptually equivalent and thus difficult to discriminate.

Data reported by Maki et al. (1979) are inconsistent with a perceptual encoding explanation for the rightleft effect. When subjects were taught to discriminate between mirror images by applying neutral verbal labels, right-left mirror images were discriminated as quickly as up-down mirror images. Results such as these led Maki et al. to conclude that a right-left effect occurs when the verbal labels "right" and "left" or "east" and "west" are used in the comparison stage of processing. In addition, their results suggested that some of the difficulty may be localized in the verbal encoding stage of processing.

The conclusion that right-left confusion has something to do with applying verbal labels to directional stimuli might be considered a special case of the theoretical position espoused by Corballis and Beale (1976). Both Farrell (1979) and Maki et al. (1979) used mirror image stimulus discrimination tasks to assess the subject's ability to tell right from left. Corballis and Beale argue that discriminating between right-left mirror images is not a perceptual problem but a memory and labeling problem. That is, the perceptual codes are not transferred homotopically, as proposed by Noble (1968), but are veridically represented in each hemisphere. Consequently, right-left information is as salient as its up-down orientation. However, when a perceptual event is encoded into memory, the memory trace laid down in one hemisphere is right-left reversed or "sym. metrized" when transferred to the other hemisphere. Thus each hemisphere contains a veridical and a rightleft reversed memory trace for a single perceptual event, and as a consequence, the right-left orientation of the event is effectively lost in the long-term memory system. This is the mechanism proposed to underlie mirror image generalization and the problem bilaterally symmetric organisms have in recognizing right-left mirror images. Integral to Corballis and Beale's theory is the supposition that any time a non-mirror image response or label is mapped onto a mirror image stimulus, right-left confusion should result. Consequently, the labels themselves are incidental to the effect, which should occur regardless of the particular label applied.

In the present study, we report a series of experiments designed to determine if the locus of the rightleft effect is in the perceptual encoding or verbal encoding stage, for tasks that are analogous to H. Clark and Chase's (1972) sentence-picture comparison task. In other words, we address the following question: Is right-left confusion attributable to difficulty in perceptually encoding direction or position in the horizontal dimension, as suggested by Farrell (1979), or is there something about the words "right" and "left" or "east" and "west" that makes them more difficult to process as claimed by Maki and colleagues (Maki, 1979b; Maki et al., 1979)?

Corballis and Beale (1976) delineate two types of tasks that test right-left confusion: left-right response differentiation and mirror image stimulus discrimination. In a left-right response differentiation task, a neutral stimulus or label is mapped onto a left or right response (e.g., a rat turns right in a $\mathrm{T}$ maze when a red light is displayed and left for a green light). In a mirror image discrimination task, right-left information is encoded into a non-mirror image response. For example, a subject who responds with the word "go" to one stimulus and refrains from responding to the other stimulus of a right-left mirror-image pair is making a mirror image discrimination.

In the first three experiments presented here, the subjects' ability to tell right from left was tested with a left-right response-differentiation task. The outcomes of these experiments demonstrate that there is something about applying verbal labels to judgments of relative location that contributes to right-left confusion. In Experiments 4-6, right-left confusion was tested with a mirror image discrimination task. In Experiment 4, we found no right-left effect in a go/no-go task. This failure to replicate Farrell's (1979) Experiment 3 dispelled any lingering reservations we may have had about rejecting the perceptual code as the locus of the effect. In conjunction with Experiment 4, Experiments 5 and 6 indicated that the labels themselves are not an incidental but a determining factor in eliciting right-left confusion in mirror image discrimination.

\section{EXPERIMENT 1}

In Experiment 1, we designed a forced-choice RT task that allowed us to independently manipulate verbal labeling and perceptual encoding. The experimental paradigm was as follows. On each trial, a circular 
array, such as shown in Figure 1, was presented. Centered within the circle was a letter (i.e., N, S, E, or W) that was flanked on either side by the numerals 1 and 2 . The subject's task was to identify the numeral lying in the compass direction specified by the letter. In one of our experimental conditions, 0-deg map axes rotation, direction was defined in accordance with the conventional map frame of reference shown in Figure 2a, with north corresponding to up, east to right, and so on. In this condition, to respond to the labels "north" and "south," subjects must perceptually encode relative location within the vertical axis and to respond to the labels "east" and "west," subjects must encode location within the horizontal axis. Hence for the stimulus in Figure 1a, west is defined as being to the left of center and the correct response would be 1 . To disentangle the effect of verbal labels from that of spatial axis, we rotated the map axes $90 \mathrm{deg}$ clockwise, as shown in Figure 2b. Now to respond to the labels "north" and "south," location within the horizontal axis must be perceptually encoded, whereas east-west judgments require encoding vertical location. Since west refers to up when map axes are rotated 90 deg clockwise, the correct response to the stimulus in Figure $1 \mathrm{~b}$ is 1 .

Orthogonal manipulation of verbal labels and spatial axes allowed us to isolate the right-left effect in either the perceptual or the verbal encoding stage of processing, given the following two assumptions. First, we assume the response-selection stage is held constant across experimental conditions, since the frequency of correct response, either 1 or 2 , is the same across all factor-level combinations. Second, we assume the comparison stage of processing is effectively bypassed in our task. $\mathrm{H}$. Clark and Chase (1972, pp. 505-506) suggest that in a forcedchoice task, spatial labels primarily serve as pointers, directing attention to the target. After identifying the target, the subject does not have to compare its location with that described by the verbal label, as in a verification task, but simply has to respond with its name.

It should be pointed out that two objections could be raised to the classification of our task as a left-right response-differentiation task. First, the verbal labels in this task could provide directional cues that would allow the subject to circumvent the requirement of telling right from left. For example, in the 0-deg rotation condition, subjects could respond to arrays containing the letter E by simply noting that the letter "points"
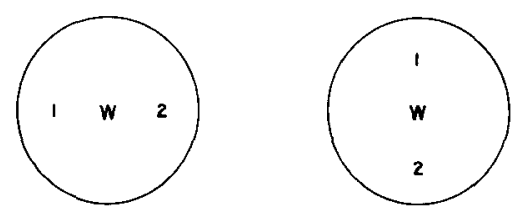

(a)

Figure 1. Example of a stimulus display in the (a) $0-\mathrm{deg}$ and (b) 90-deg rotation conditions of Experiment 1.

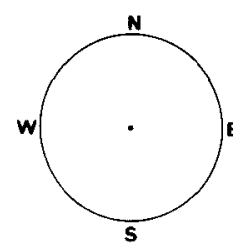

(a)

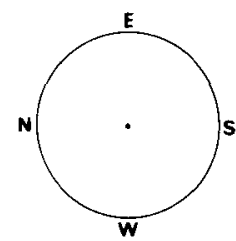

(c)

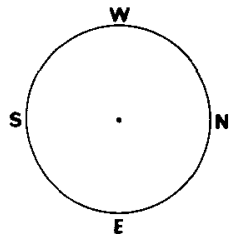

(b)

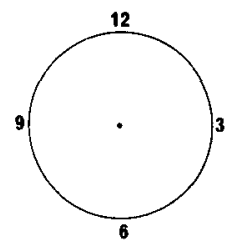

(d)
Figure 2. Representations of schematic maps defining the orientation of the compass directions in the (a) $0-\mathrm{deg}$, (b) 90-deg, and (c) 270-deg rotation conditions in Experiments 1 and 2 and (d) schematic clock defining direction in Experiment 3. (From Sholl \& Egeth, in press. Copyright 1980 by Plenum Press. Reprinted by permission.)

toward the correct location. Since the presence of an asymmetrical cue in the array should attenuate the right-left effect, we do not consider this feature of the task to be particularly problematic. Second, the task does not require an overt directional response (e.g., turning to the right, raising the right hand). However, we propose that the task does require a covert orienting response, in that attention moves in an analog fashion across the visual field to the location of the target numeral (e.g., Shulman, Remington, \& McLean, 1979).

If the locus of the right-left effect is in the verbal labeling process, we would expect north-south judgments to be faster than east-west in both the 0-deg and 90-deg rotation conditions, even though in the 90-deg condition, north and south describe location on the horizontal axis and east and west describe vertical location. Statistically, this prediction translates to a main effect of verbal labels. Alternatively, if the difficulty of perceptually encoding location on the horizontal axis is the cause of right-left confusion, we would expect east-west judgments to be slower in the 0-deg than the 90-deg condition and north-south judgments to be slower in the 90-deg than the 0-deg condition. Hence a perceptual encoding explanation corresponds statistically to a disordinal interaction between rotation and verbal label. If both the verbal and perceptual encoding stages contribute additively to the effect, as might be expected given H. Clark and Chase's (1972) theoretical position that verbal and perceptual codes have similar proposiional formats, the above two predictions should both be realized. That is, north-south judgments would always be faster than east-west judgments, and north-south judgments would be faster in the 0-deg than the 90-deg condition, whereas east-west judgments would be faster in the 90-deg than in the 0-deg condition; this 
statistically translates into both a main effect of verbal labels and a Verbal Label by Rotation interaction.'

\section{Method}

Subjects. Twelve Johns Hopkins University students served as subjects in this experiment. Two of the 12 subjects were replaced, one because of experimenter error and one because of an abnormally high average error rate of $13 \%$. (Error rates for the subjects included in the analysis ranged from $1 \%$ to $5.6 \%$.) Subjects were either paid for their participation or received credits to be applied toward psychology course requirements. All subjects across all experiments reported normal or corrected-to-normal vision.

Stimulus materials. Two decks of 32 cards were prepared for each rotation condition. A circle with a diameter of $70 \mathrm{~mm}$ was centered on each card. An uppercase N, E, S, or W was printed at the center of the circle. Each letter was horizontally or vertically flanked by the numerals 1 and 2 . The numerals were positioned $19 \mathrm{~mm}$ from the center of the circle. The letters were black Futura demibold 24-point Chartpak, and the numerals were black Simplex bold 8-point Chartpak. Within each deck of 32 cards, there were four replicas of each of 8 basic cards. There were two basic cards for each compass letter. On one card, the correct response was " 1 "; on the other card, the positions of the numerals 1 and 2 were reversed, and so the correct response was " 2 ." Stimulus cards were presented in a Gerbrands two-field mirror tachistoscope at a viewing distance of $59 \mathrm{~cm}$. The diameter of the circle spanned $6.8 \mathrm{deg}$ of visual angle, and the distance from the center of the circle to the center of a numeral subtended $1.75 \mathrm{deg}$ of visual angle.

Design. A mixed experimental design was utilized in this study. The between-subjects factor was the order in which subjects received the rotation conditions. Six subjects were randomly assigned to the $0-$ to $90-\mathrm{deg}$ order, and the other six subjects were assigned to the 90- to 0-deg order. Each experimental session was devoted entirely to a single rotation condition. Every subject participated in two $30-\mathrm{min}$ sessions that were separated by approximately 1 week.

Each experimental session was divided into four blocks of trials. Within a block of 32 trials, the four cardinal directions were each tested eight times. Separate decks of cards were used in Blocks 1 and 2. The sequence of cards in each deck was randomized for each subject, with the restriction that no letter occur more than three times consecutively. The sequence of cards in Block 1 was reversed in Block 3 , and the sequence in Block 2 was reversed in Block 4. The first block of trials served as practice in both experimental sessions. Aside from blocks $(2,3,4)$, the within-subjects variables were rotation condition (0 deg, $90 \mathrm{deg}$ ) and verbal labels (north, south, east, west).

Procedure. At the beginning of each rotation condition, the subject was (1) read a set of instructions to familiarize him or her with the experimental task and (2) shown a schematic map (as in Figure 2) identifying the orientation of the compass directions for that session.

Immediately preceding each trial, a warning tone served as a cue for the subject to focus on the fixation point. Stimulus duration was fixed at $200 \mathrm{msec}$, and a fixation field was present whenever the stimulus array was off. Subjects were instructed to respond as quickly as possible and within that constraint to be accurate. Vocal RT was measured to the nearest $1 \mathrm{msec}$. Intertrial interval was approximately $10 \mathrm{sec}$, and there was a short break between Blocks 2 and 3 .

If subjects made an error on any trial, the next trial was an unannounced practice trial. Special "error" cards were made up for this purpose, and RT was not recorded on these trials. An arbitrary criterion of $1,500 \mathrm{msec}$ was set as a ceiling for acceptable response time. This cutoff resulted in the elimination of $.17 \%$ of the trials ( 4 trials of a total of 2,304 ). Mean RT from each factor-level combination was based on each subject's correct responses. If the verbal response failed to trigger the voice-activated relay, the trial was discarded.

\section{Results}

Mean latencies and error rates are presented in Table 1. The effect of verbal labels was significant $[F(3,30)=13.69, p<.001]$. When averaged over rotation conditions, responses to the letters indicating north and south averaged $51 \mathrm{msec}$ faster than those to east and west. A post hoc Scheffe comparison indicated this difference was significant. Overall, it took subjects $19.5 \mathrm{msec}$ longer to perceptually encode location in the vertical than in the horizontal dimension. This result is unexpected and appears to be due primarily to the long response latencies in the east-west/90-deg factorlevel combination. However, the effect is not statistically reliable, since the Rotation Condition by Verbal Label interaction was not significant $[F(3,30)=2.71, p>.05]$.

On the average, subjects responded a significant $32 \mathrm{msec}$ faster in the 0 -deg than in the 90-deg rotation condition $[F(1,10)=15.32, p<.005]$. There were no other significant effects in the analysis.

\section{Discussion}

The results are supportive of a verbal encoding explanation for the right-left effect. A latency advantage for the labels "north" and "south" was obtainable regardless of whether these labels defined location on the horizontal or vertical spatial axis. A perceptual encoding explanation for the right-left effect predicts horizontal judgments should take longer than vertical judgments in both the 0-deg and the 90-deg rotation conditions. However, this prediction was not supported if anything, there was a tendency for vertical judgments to take longer than horizontal judgments.

If the effects observed in this experiment are valid they should be obtained when map axes are rotatec 270 deg clockwise, as shown in Figure 2c. In this rota

Table 1

Mean Latencies (RT) and Proportion of Errors (PE) for Locational Judgments in Experiment 1 as a Function of Rotation Condition, Spatial Axis, and Verbal Label

\begin{tabular}{|c|c|c|c|c|c|}
\hline \multirow{2}{*}{$\begin{array}{l}\text { Spatial } \\
\text { Axis }\end{array}$} & \multirow{2}{*}{$\begin{array}{l}\text { Verbal } \\
\text { Label }\end{array}$} & \multicolumn{2}{|c|}{ Label } & \multirow[b]{2}{*}{ Axis } & \multirow{2}{*}{$\begin{array}{c}\text { Rota- } \\
\text { tion }\end{array}$} \\
\hline & & RT & $\mathrm{PE}$ & & \\
\hline & & \multicolumn{4}{|c|}{ 0-Deg Rotation Condition } \\
\hline Vertical & $\begin{array}{l}\text { North } \\
\text { South }\end{array}$ & $\begin{array}{l}727 \\
743\end{array}$ & $\begin{array}{l}.007 \\
.020\end{array}$ & 735 & \multirow{2}{*}{751} \\
\hline \multirow[t]{2}{*}{ Horizontal } & $\begin{array}{l}\text { East } \\
\text { West }\end{array}$ & $\begin{array}{l}763 \\
771\end{array}$ & $\begin{array}{l}.038 \\
.017\end{array}$ & 767 & \\
\hline & & \multicolumn{4}{|c|}{ 90-Deg Rotation Condition } \\
\hline Horizontal & $\begin{array}{l}\text { North } \\
\text { South }\end{array}$ & $\begin{array}{l}732 \\
764\end{array}$ & $\begin{array}{l}.007 \\
.017\end{array}$ & 748 & \multirow{2}{*}{783} \\
\hline Vertical & $\begin{array}{l}\text { East } \\
\text { West }\end{array}$ & $\begin{array}{l}831 \\
806\end{array}$ & $\begin{array}{l}.050 \\
.033\end{array}$ & 819 & \\
\hline
\end{tabular}


tion condition, north and south again refer to location on the horizontal spatial axis, whereas east and west refer to location on the vertical axis. Experiment 2 tests this expectation.

\section{EXPERIMENT 2}

\section{Method}

The method followed in Experiment 2 was for the most part identical to that outlined in Experiment 1. Twelve new subjects, drawn from the same subject population, were employed. The 270 -deg rotation condition was introduced by the schematic map shown in Figure 2c. An arbitrary criteria of $2,000 \mathrm{msec}$ was set as a limit on acceptable RT. This criterion resulted in the elimination of 1 trial of a total of 2,304. (One subject consistently responded with latencies over $1,500 \mathrm{msec}$, so we extended the criteria to $2,000 \mathrm{msec}$ in this experiment.)

\section{Results}

Mean latencies and error rates are listed in Table 2. Once again, there was a main effect of verbal labels $[F(3,30)=11.08, p<.001]$. A Scheffe post hoc comparison showed that the $60.5 \mathrm{msec}$ advantage of north and south over east and west was significant. The only other significant effect was that of blocks $[F(2,20)=$ $4.00, \mathrm{p}<.05]$, indicating that latencies decreased with practice.

\section{Discussion}

The outcome of Experiment 2 is consistent with Experiment 1. A significant advantage of the labels

Table 2

Mean Latencies (RT) and Proportion of Errors (PE) for Locational Judgments in Experiment 2 as a Function of Rotation Condition, Spatial Axis, and Verbal Label

\begin{tabular}{|c|c|c|c|c|c|}
\hline \multirow{2}{*}{$\begin{array}{l}\text { Spatial } \\
\text { Axis }\end{array}$} & \multirow{2}{*}{$\begin{array}{l}\text { Verbal } \\
\text { Label }\end{array}$} & \multicolumn{2}{|c|}{ Label } & \multirow[b]{2}{*}{ Axis } & \multirow{2}{*}{$\begin{array}{c}\text { Rota- } \\
\text { tion }\end{array}$} \\
\hline & & RT & PE & & \\
\hline & & \multicolumn{4}{|c|}{ 0-Deg Rotation Condition } \\
\hline Vertical & $\begin{array}{l}\text { North } \\
\text { South }\end{array}$ & $\begin{array}{l}756 \\
784\end{array}$ & $\begin{array}{l}.027 \\
.030\end{array}$ & 770 & \multirow{2}{*}{798} \\
\hline \multirow[t]{2}{*}{ Horizontal } & $\begin{array}{l}\text { East } \\
\text { West }\end{array}$ & $\begin{array}{l}831 \\
819\end{array}$ & $\begin{array}{l}.027 \\
.024\end{array}$ & 825 & \\
\hline & & \multicolumn{4}{|c|}{ 270-Deg Rotation Condition } \\
\hline Horizontal & $\begin{array}{l}\text { North } \\
\text { South }\end{array}$ & $\begin{array}{l}768 \\
795\end{array}$ & $\begin{array}{l}.017 \\
.031\end{array}$ & 782 & \multirow{2}{*}{815} \\
\hline Vertical & $\begin{array}{l}\text { East } \\
\text { West }\end{array}$ & $\begin{array}{l}824 \\
871\end{array}$ & $\begin{array}{l}.030 \\
.068\end{array}$ & 848 & \\
\hline
\end{tabular}

"north" and "south" in both the 0-deg and 270-deg rotation conditions supports a verbal encoding explanation for right-left confusion in the adult.

The results of the first two experiments can be clarified with a simple descriptive model. The model is based on the assumption that RT can be partitioned into a sequence of additive component-process durations (Sternberg, 1969). Combining the data from Experiments 1 and 2 allowed us to estimate three parameters and two base times (one base time for each experiment). Parameter a represents the extra time it takes to process the words "east" and "west" in the verbal encoding stage of the sequence. Parameter $b$ is the extent to which time to perceptually encode location on the horizontal dimension exceeds time to encode location on the vertical dimension. The increment in RT caused by rotating the map axes into a novel orientation is estimated by Parameter $\mathrm{c}$.

The parameters composing mean RT for each of the four factor-level combinations of Experiments 1 and 2 are listed at the center of Table 3. Parameters were estimated from the eight latencies listed at the left of Table 3. After making the appropriate subtractions, the two base times and three parameters were estimated at $t_{1}=733 \mathrm{msec}, t_{2}=772 \mathrm{msec}, \quad a=56 \mathrm{msec}, \quad b=$ $-12.5 \mathrm{msec}$, and $\mathrm{c}=25 \mathrm{msec}$. The preceding estimates left 3 of 8 degrees of freedom for error and a root mean squared deviation equal to $5.6 \mathrm{msec}$. As can be observed from examining Table 3, the predicted latencies, fairly closely approximate the observed latencies, and, as a consequence, the model accounts for $98 \%$ of the variance in the observed latencies. An F test for goodness of fit (Miller \& Greeno, 1978) indicated that the residual variance was not significantly greater than would be expected by chance $(F<1)$.

On the average, then, east-west judgments took $56 \mathrm{msec}$ longer than north-south judgments. This rightleft effect was significant in both Experiments 1 and 2 . Contrary to our initial expectations, there was a nonsignificant trend toward longer latencies for locating vertically aligned objects. Although overall latencies were longer when map axes were rotated, this effect was signiticant only in Experiment 1 . Theoretically, to what the effect of the rotation of map axes should be attributed is not exactly clear. One possibility is that Parameter $c$ represents mental rotation time. This possibility could be tested with a 180-deg rotation condition.

Table 3

Observed Latencies, Descriptive Model, and Predicted Latencies for Experiments 1 and 2

\begin{tabular}{|c|c|c|c|c|c|c|}
\hline \multirow{2}{*}{$\begin{array}{l}\text { Factor-Level } \\
\text { Combination }\end{array}$} & \multicolumn{2}{|c|}{ Observed Latencies } & \multicolumn{2}{|c|}{ Descriptive Model } & \multicolumn{2}{|c|}{ Predicted Latencies } \\
\hline & Experiment 1 & Experiment 2 & Experiment 1 & Experiment 2 & Experiment 1 & Experiment 2 \\
\hline & \multicolumn{6}{|c|}{ 0-Deg Rotation Condition } \\
\hline \multirow[t]{2}{*}{$\begin{array}{l}N / S \\
E / W\end{array}$} & $\begin{array}{l}735 \\
767\end{array}$ & $\begin{array}{l}770 \\
825\end{array}$ & $\begin{array}{l}t_{1} \\
t_{1}+a+b\end{array}$ & $t_{2}+a+b$ & $\begin{array}{l}733.0 \\
776.5\end{array}$ & $\begin{array}{l}772.0 \\
815.5\end{array}$ \\
\hline & \multicolumn{6}{|c|}{ Rotated Condition } \\
\hline $\begin{array}{l}N / S \\
E / W\end{array}$ & $\begin{array}{l}748 \\
819 \\
\end{array}$ & $\begin{array}{l}782 \\
848 \\
\end{array}$ & $\begin{array}{l}t_{1}+b+c \\
t_{1}+a+c\end{array}$ & $\begin{array}{l}t_{2}+b+c \\
t_{2}+a+c\end{array}$ & $\begin{array}{l}745.5 \\
814.0\end{array}$ & $\begin{array}{l}784.5 \\
853.0\end{array}$ \\
\hline
\end{tabular}




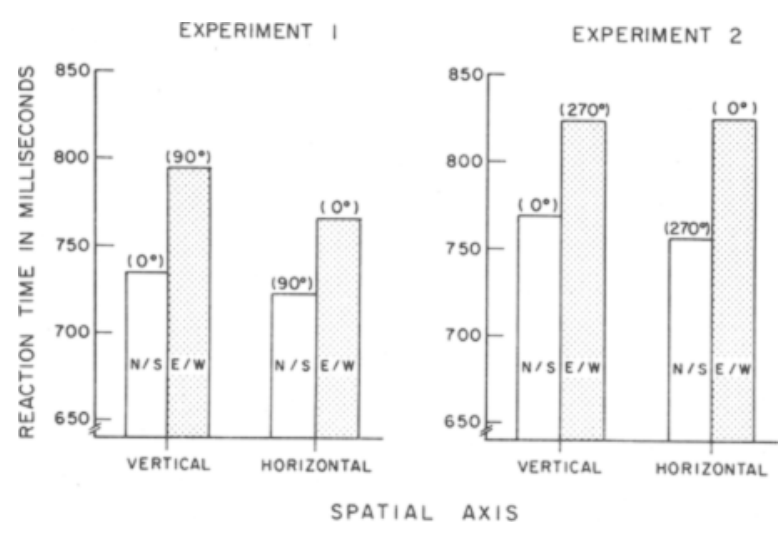

Figure 3. Mean RT for Experiments 1 and 2 as a function of verbal labels and spatial axis with the estimated 25 -msec rotation time subtracted from the 90-deg and 270-deg data points. (From Sholl \& Egeth, in press. Copyright 1980 by Plenum Press. Reprinted by permission.)

If $c$ represents mental rotation time, the value of $c$ should increase with rotation angle, so that it would be approximately twice as large (i.e., $50 \mathrm{msec}$ ) at $180 \mathrm{deg}$ as at $90 \mathrm{deg}$ or $270 \mathrm{deg}$ (Cooper \& Shepard, 1973).

To determine if $c$ is mental rotation time, 10 additional subjects were run in a 0 -deg and in a 180-deg rotation condition (order counterbalanced across sessions). The 180-deg condition proved much more difficult than the 90- and 270-deg conditions. With regard to accuracy, the average error rate in the 180-deg rotation condition was $8.7 \%$, a value considerably larger than the $3.1 \%$ average error rate in the 90 - and 270 -deg conditions and the $2.7 \%$ average error rate in the 0 -deg rotation conditions. Furthermore, four of the subjects in the 180-deg condition responded with an error rate greater than $10 \%$. In both the $90-$ and $270-$ deg rotation conditions, there was only one subject with an error rate above $10 \%$. This subject also responded with an error rate above $10 \%$ in the simpler 0-deg condition, and he was replaced because of apparent difficulty with the task. We decided not to replace subjects in the present experiment when it became clear that a high proportion of subjects were having trouble with the 180 -deg condition. With regard to time, the value of c (which equals the mean difference in RT between the 0 - and 180-deg conditions) was $131 \mathrm{msec}$, which is much greater than the expected value of $50 \mathrm{msec}$. These results suggest that $c$ does not represent simple mental rotation time.

A remaining possibility is that $c$ represents the extra time it takes to remember relatively unfamiliar propositions (or associations) such as "south is up" or "east is left." Of course, there are novel propositions to be remembered in the 90- and 270-deg conditions, as well. To speculate for a moment, what makes the 180-deg condition so difficult may be the congruence of the spatial axes in both the 0 - and 180-deg conditions (i.e., the north-south axis is still vertical in the 180-deg condition, but inverted). Consequently, the instructions to respond to map axes rotated 180 deg may have come into conflict with the subject's propensity to respond in accordance with his or her long-term association of north with up, east with right, and so on. In the 90and 270-deg rotation conditions, the north-south spatial axis was horizontal. Hence it may have been more readily apparent that the long-term associations of north with up, and so on, were inapplicable in these rotation conditions. Regardless of how one interprets $c$, perhaps the clearest depiction of the results of Experiments 1 and 2 emerges when $c$ is subtracted from the means in the 90- and 270-deg conditions, as shown in Figure 3.

Predicated on the outcome of Experiments 1 and 2, it seems that the usual right-left effect demonstrated in adults is due more to the presence of the verbal labels "east" and "west" than to any deficiency in judging location in the horizontal dimension. If this conclusion is correct, then there should be no analog of the right-left effect if a reference system with neutral labels is used. To this end, in Experiment 3, we used circular displays again, but the circles represented a schematic clock, as shown in Figure 2d.

\section{EXPERIMENT 3}

The paradigm employed in Experiment 3 was identical to that used in Experiments 1 and 2. Vertical direction was defined by the numerals 12 and 6 , whereas direction within the horizontal axis was defined by the numerals 3 and 9 . The numerals were flanked by the letters $x$ and $o$, as shown in Figure 4, and the subject's task was to identify the letter lying in the "direction" specified by the numeral. This task, which does not require any overt encoding of directional labels, was designed to provide converging evidence that the right-left effect is a labeling phenomenon. Because of their association with the face of a clock, the numerals 1 through 12 do convey spatial information, but they are seldom used in the course of everyday conversation to describe spatial location. This probably places them on a continuum someplace between truly directional labels (e.g., north, south, east, and west) and arbitrary labels (e.g., F, J, $X$, and Q), such as those employed by Maki et al. (1979). If perceptual discrimination of relative location is more difficult within the horizontal dimension, the results should be consistent with Farrell's (1979, Experiment 3) results, and 3-9 judgments should take longer than
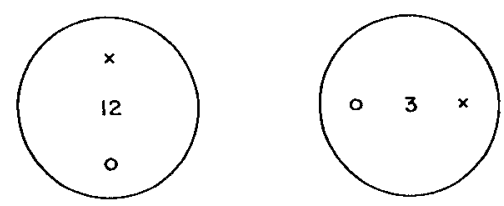

Figure 4. Examples of the 12 and 3 stimulus displays used in Experiment 3. 
12-6 judgments. Alternatively, if we are correct in concluding that directional verbal labels cause the right-left effect, then there should be no advantage of 12-6 over $3-9$ judgments.

\section{Method}

Subjects. Twelve new subjects were drawn from the same subject pool.

Stimulus materials. Two decks of 32 cards each were prepared for this experiment. A 70-mm circle was drawn on each card. A 12,3,6, or 9 was drawn in black ink at the center of each circle. Positioned $19 \mathrm{~mm}$ to either side of the numeral were the letters $x$ and $o$. When the numerals were 12 and 6 , the letters were aligned vertically, and when the numerals were 3 and 6 , the letters were horizontally aligned, as shown in Figure 4. Alphanumerics were drawn with a Pickett 13 stencil. The numerals were $6.4 \mathrm{~mm}$ high and the letters were $4.7 \mathrm{~mm}$ high. Within each deck of 32 cards, there were four instances of each of 8 basic cards. There were two basic cards for each numeral, the difference between the cards being that the position of $\mathrm{x}$ and $\mathrm{o}$ were reversed in one card relative to the other. As in the previous two experiments, the stimulus cards were shown in a Gerbrands two-field mirror tachistoscope. At a viewing distance of $59 \mathrm{~cm}$, the diameter of the circle subtended $6.8 \mathrm{deg}$ of visual angle, and the center-to-center distance between the numeral and each letter subtended $1.75 \mathrm{deg}$ of visual angle.

Procedure. The procedure was essentially the same as that followed in Experiments 1 and 2. Each subject was initially shown a schematic clock to familiarize him or her with how the numerals were to be used as locatives. Subjects were run through four blocks of trials with 32 trials/block. The first block of trials was considered practice; the last three biocks of trials were experimental trials. Each of the two decks of cards was put in a different random order for each subject, with the restriction that no numeral appear more than three times in a row. Blocks 3 and 4 were simply the sequence of cards in Blocks 1 and 2 reversed. The experiment was run in one session that lasted approximately $30 \mathrm{~min}$. In all other respects, the procedure was identical to Experiment 1. A 1,500msec RT ceiling resulted in the elimination of 3 trials of a total of 1,152 .

Design. In this and all subsequent experiments, the verbal label (or in Experiment 4, directional judgment) means were contrasted with planned orthogonal comparisons. The question of primary import was whether response to horizontal labels was slower than response to vertical labels. Second, we were interested in ascertaining if responses to labels denoting up or above were responded to faster than labels denoting down or below. Third, we contrasted labels denoting right with those denoting left.

\section{Results}

Mean RTs and error rates are depicted in Table 4. The 14-msec advantage of 3-9 over 12-6 judgments was not significant $[F(1,33)=1.90, p>.10]$. Within dimensions, both the $31-\mathrm{msec}$ advantage of 12 over $6[\mathrm{~F}(1,33)=$ $4.46, p<.05]$ and the $47-\mathrm{msec}$ advantage of 3 over $9[\mathrm{~F}(1,33)=10.69, \mathrm{p}<.001]$ were significant.

\section{Discussion}

The results of Experiment 3 provide strong converging evidence that, in our task, the locus of the right-left effect is in the verbal encoding stage of processing. When the numerals $12,6,3,9$ are substituted for the letters $\mathrm{N}, \mathrm{S}, \mathrm{E}, \mathrm{W}$ in equivalent paradigms, locating objects in
Table 4

Mean Latencies (RT) and Proportion of Errors (PE) for Locational Judgments as a Function of Clock Numerals in Experiment 3

\begin{tabular}{|c|c|c|c|c|}
\hline \multirow{2}{*}{$\begin{array}{c}\text { Spatial } \\
\text { Axis }\end{array}$} & \multirow{2}{*}{$\begin{array}{c}\text { Clock } \\
\text { Numeral }\end{array}$} & \multicolumn{2}{|c|}{ Numeral } & \multirow[b]{2}{*}{ Axis } \\
\hline & & RT & $\mathrm{PE}$ & \\
\hline Vertical & $\begin{array}{r}12 \\
6\end{array}$ & $\begin{array}{l}742 \\
773\end{array}$ & $\begin{array}{l}.00 \\
.03\end{array}$ & 757.5 \\
\hline Horizontal & $\begin{array}{l}3 \\
9\end{array}$ & $\begin{array}{l}720 \\
767\end{array}$ & $\begin{array}{l}.01 \\
.02\end{array}$ & 743.5 \\
\hline
\end{tabular}

the vertical axis takes on the average $14 \mathrm{msec}$ longer than locating objects in the horizontal axis. Although this result is not significant, it is consistent with Parameter $b$, which was estimated from the combined results of Experiments 1 and 2 to be $-12.5 \mathrm{msec}$. These results suggest that it does not necessarily take longer to locate objects within the horizontal dimension than to locate objects within the vertical dimension.

\section{EXPERIMENT 4}

We were troubled at this point by the results of Farrell's (1979) Experiment 3, in which he found a right-left effect in a mirror image discrimination task even though subjects did not use directional verbal labels. Farrell's results are clearly inconsistent with the data reported thus far. One possible source of discrepancy is the task. Our task has been a responsedifferentiation task, whereas Farrell's task was a mirror image discrimination task. It is possible that there may be something unique to mirror image discrimination that impedes the perceptual encoding of horizontally aligned stimuli. Alternatively, some subtle experimental detail may account for Farrell's results. In accordance with this latter possibility, there was one aspect of Farrell's procedure that seemed problematic. Recall that in Farrell's procedure, trials were blocked such that in each block subjects saw only right and left or up and down arrows, a single arrow being presented on each trial. Within a block, subjects made a buttonpressing response to the arrow designated as the target. To control for stimulus-response compatibility effects, subjects were instructed to respond by pressing two buttons simultaneously, one with the left hand and one with the right hand. Since the buttons were horizontally aligned, the requirement to respond simultaneously may have inadvertently generated some sort of interference when response was to the horizontal arrows, thereby increasing response time in that condition relative to the vertical arrows. In Experiment 4, we eliminated that problem by replicating Farrell's experiment using a vocal rather than a manual response. Subjects responded with the word "go" to the onset of the target arrow. 


\section{Method}

Subjects. Twelve subjects were drawn from a summer subject pool and were paid for their participation in the experiment.

Procedure. Stimuli were arrowheads (e.g., $>$ ) drawn in black ink with a Pickett 1311 stencil. The arrows were centered on a fixation point that was present at all times except during stimulus presentation. Each side of the arrow measured $16 \mathrm{~mm}$, and at a viewing distance of $91 \mathrm{~cm}$, each side was $1 \mathrm{deg}$ of visual angle in length.

Subjects received four blocks of trials in counterbalanced order. Only right-left or up-down arrows appeared within a single block. At the beginning of each block of trials, subjects were instructed to make the verbal response "go" to one member of the pair and to refrain from responding to the other. Three subjects were randomly assigned to each of the following response sequences: (1) down, up, right, left; (2) up, down, left, right; (3) right, left, down, up; (4) left, right, up, down. This generated a counterbalanced design that was analyzed as a Latin square. Stimuli were randomly sequenced within each block of 52 trials, with the restriction that no stimulus appear more than three times consecutively. Each experimental block was preceded by 20 practice trials. At the beginning of each experimental session, a set of instructions was read to the subject. Subjects were instructed to respond as quickly as possible and, given that instruction, to be accurate. At the start of each trial, a warning tone preceded stimulus onset by $500 \mathrm{msec}$, serving as a signal for the subject to focus on the fixation point. Stimulus duration was $100 \mathrm{msec}$, and the intertrial interval was $3.8 \mathrm{sec}$. Vocal RT was measured to the nearest $1 \mathrm{msec}$ from stimulus onset. The above sequence of events was controlled by an ICONIX system of solid state logic. Subjects were given a 1-min rest between Blocks 2 and 3 . The entire session lasted about $30 \mathrm{~min}$.

\section{Results}

Mean RTs as a function of arrow direction are shown in Table 5. An analysis of variance for a replicated Latin square was carried out on the data to generate the appropriate error term for the planned comparisons. The contrasts substantiated the obvious: Right-left arrows were discriminated as quickly as up-down arrows, and there were no latency differences within dimensions.

A significant practice effect $[F(3,30)=4.66, p<.01]$ reflected an improvement in $\mathrm{RT}$ following the first block of trials.

\section{Discussion}

Our finding replicates a study by Maki (1979b) that appeared in print after we had completed this experiment. Using the vocal response "yes" and essentially the same procedure, Maki also reported no effect of arrow direction. Hence, we can only conclude that Farrell's (1979) results were the result of a Type I error

Table 5

Mean Latencies (RT) and Proportion of Errors (PE) for Directional Judgments in Experiment 4

\begin{tabular}{cll}
\hline Direction & RT & PE \\
\hline$\wedge$ & 421 & .005 \\
$\bigvee$ & 425 & .003 \\
$>$ & 425 & .006 \\
$<$ & 420 & .002 \\
\hline
\end{tabular}

or stimulus-response incompatibility. The perceptual identification of a mirror image stimulus is apparently no harder for adults if the stimulus is a right-left mirror image than if it is an up-down mirror image.

\section{EXPERIMENT 5}

If using the compass words in a spatial discrimination task is sufficient to cause right-left confusion, we would expect a right-left effect in a go/no-go task when subjects respond with the appropriate compass word to a directional arrow. Experiment 5 directly tested this expectation.

\section{Method}

The method used in Experiment 5 was generally the same as that used in Experiment 4 , with the following exceptions. Twenty subjects were employed in this experiment. These subjects were drawn from the subject pool in use during the academic year.

Two modifications were made in the procedure. First, in this experiment, subjects responded to arrows pointing up, down, right, or left with the words "north," "south," "east," and "west," respectively. That is, within each block of trials, rather than responding with the word "go" to the target arrow, subjects responded with the appropriate compass term. In all other respects, the task was exactly the same as in Experiment 4. Only right and left or up and down arrows appeared within a given block, and in each block subjects labeled only the target arrow. The order in which arrows were designated as targets was counterbalanced in the same Latin square outlined in Experiment 4.

The second modification was necessitated by the fact that for the first time in this series of experiments, subjects responded vocally with the compass directions themselves. As it is possible that these words differ from each other in their articulatory characteristics, voice onset times (VOTs) were estimated as follows. At the end of the experimental session, subjects were given four blocks of 26 trials. On each trial, a dot appeared at the center of an otherwise blank fixation field. Stimulus duration was $100 \mathrm{msec}$ and occurred $500 \mathrm{msec}$ after a warning tone. The subject's task was simply to respond with the appropriate compass word as soon as he or she saw the dot. Two sequences of trials were prepared. One sequence was labeled either Deck A or Deck D and the other sequence was labeled either Deck B or Deck C. Subjects responded "north" to the cards in Deck A, "sou th" to Deck B, "east" to Deck C, and "west" to Deck D. In each deck, six of the trials were catch trials, randomly interspersed within the sequence to guard against anticipatory errors. The decks were concatenated in the same order the subject received the experimental trials. RT was measured to the nearest $1 \mathrm{msec}$ from stimulus onset to vocal response.

\section{Results}

Mean RT for directional judgments and VOT are listed in Table 6. VOTs for the compass words were significantly different from each other $[F(3,54)=15.49$, $p<.001]$. Hence in order to obtain latencies for directional judgments that were not confounded with VOTs, differences between mean VOTs and directional judgments were computed across conditions for each subject. In using this subtractive procedure, we are making the assumption that VOT simply adds a constant amount to RT in the primary task. The difference 
Table 6

Mean Vocal Onset Times (VOT), Mean Directional Judgment Times (T) and Proportion of Errors (PE), and Difference Scores (DS) in Experiment 5

\begin{tabular}{|c|c|c|c|c|c|}
\hline \multirow[b]{2}{*}{ Direction } & \multirow[b]{2}{*}{ VOT } & \multicolumn{2}{|c|}{ Directional Judgment } & \multirow[b]{2}{*}{ DS } & \multirow{2}{*}{$\begin{array}{l}\text { Dimension } \\
\text { Mean (DS) }\end{array}$} \\
\hline & & $\mathrm{T}$ & PE & & \\
\hline North & 383 & 424 & .003 & 41 & 46.5 \\
\hline South & 402 & 454 & .003 & 52 & 40.5 \\
\hline East & 406 & 467 & .001 & 61 & 575 \\
\hline West & 364 & 418 & .001 & 54 & 37.5 \\
\hline
\end{tabular}

Note-Each difference score is the VOT mean subtracted from the directional judgment mean.

scores were then analyzed with an analysis of variance for a replicated Latin square. A planned comparison indicated that the 11 -msec advantage of north-south over east-west did not quite reach significance $[F(1,54)$ $=3.01, \mathrm{p}<.10]$. Neither the $11-\mathrm{msec}$ difference between north and south nor the $7-\mathrm{msec}$ difference between west and east was significant.

\section{Discussion}

The absence of a clear up-down advantage in Experiment 5 suggests that directional verbal labels are necessary but may not be sufficient to elicit a right-left effect. Why do we get a right-left effect in Experiments 1 and 2 but no clear effect in Experiment 5? One possible difference between the experimental tasks, aside from the distinction between response differentiation and stimulus discrimination, is the extent to which a covert orienting system (e.g., Posner, 1978) may be engaged in task performance. In order to respond appropriately in the response-differentiation task employed in Experiments 1 and 2, the subject must redirect attention to a spatial location on each trial. However, in the mirror image discrimination task employed in Experiment 5, subjects might choose a strategy of attending to a single location throughout a block of trials. For example, to respond to an arrow pointing up, the subjects would merely have to look for the tip of the arrow to appear above the fixation point. Hence, we tentatively conclude that the compass terms may elicit a right-left effect only when the task requires mapping compass terms onto an egocentric spatial reference system wherein attention can be deployed up-down, right-left, or front-back. This hypothesis would predict a right-left effect in a fourstimulus/four-response mirror image discrimination task; that is, subjects would respond "north" to up arrows, "east" to right arrows, and so on, within the same block of trials. A right-left effect has in fact been found in such a task with the labels "up," "down," "right," and "left" (Farrell, 1979, Experiment 4).

An implicit assumption underlying Experiments 1.5 has been that the compass labels "north," "south," "east," and "west" are cognitively equivalent to the egocentric labels "up," "down," "right," and "left." Consequently, it was our expectation that no right-left effect should be observed if the egocentric labels were substituted in the same go/no-go discrimination task used in Experiments 4 and 5.

\section{EXPERIMENT 6}

\section{Method}

Subjects were 12 Johns Hopkins University students who either were paid for their participation in the experiment or received research credit.

The method was the same as that used in Experiments 4 and 5 , with the following two exceptions. First, the words "up," "down," "right," and "left" were used as labels for the directional arrows in the go/no-go task. Second, the VOT means were based on 10 trials. $^{2}$

\section{Results}

Table 7 gives mean VOT and mean RT for directional judgments. Since there were no significant differences among the VOT means $[F(3,30)=1.77]$, the subtractive procedure was not applied to these data. The $22.5-\mathrm{msec}$ advantage of up-down over right-left was significant $[F(1,30)=12.05, p<.001]$. There was also a $23-\mathrm{msec}$ latency advantage of up over down arrows $[F(1,30)=$ $12.05, \mathrm{p}<.001]$, but the difference between right and left arrows was not significant.

The only other significant effect was a practice effect $[F(3,23)=9.25, p<.001]$, indicating that, on the average, latencies in Block 1 were longer than those in Blocks 2-4.

\section{Discussion}

Contrary to expectation, the outcome of Experiment 6 demonstrates a right-left effect when the labels "up," "down," "right," and "left" are used to describe arrow direction. It is not entirely clear what to make of the difference in outcomes between Experiments 5 and 6. In Experiment 5, there was an 11 -msec advantage for north-south over east-west $(\mathrm{p}<.10)$. In Experiment 6 there was a $22.5 \mathrm{msec}$ advantage of up-down over right-left $(p<.001)$. It is quite possible that both effects are small but real and that the difference is due to sampling error. (In this connection, note especially that the analysis of Experiment 5 is based on difference scores, with their attendant large variability.)

Alternatively, it is possible that the difference between the experiments is as it appears to be: The

Table 7

Mean Vocal Onset Times (VOT) and Mean Directional Judgment Times (T) and Proportion of Errors (PE) in Experiment 6

\begin{tabular}{|c|c|c|c|c|}
\hline \multirow[b]{2}{*}{ Direction } & \multirow[b]{2}{*}{ VOT } & \multicolumn{2}{|c|}{ Directional Judgment } & \multirow[b]{2}{*}{ Dimension } \\
\hline & & $\mathrm{T}$ & PE & \\
\hline $\mathrm{Up}$ & 340 & 378 & .000 & 389.5 \\
\hline Down & 362 & 401 & .005 & 389.3 \\
\hline Right & 358 & 414 & .000 & 4120 \\
\hline Left & 352 & 410 & .011 & 412.0 \\
\hline
\end{tabular}


compass words yield no right-left effect, but the egocentric labels do. What might such a difference mean?

For one thing, it casts doubt on our assumption of cognitive equivalence between the two sets of labels. Such a lack of equivalence might be attributable to the different frames of reference delineated by the two sets of terms. The compass terms describe an absolute frame of reference that is fixed in space, whereas the egocentric terms describe a relative frame of reference that changes with the position of the observer. Hence discriminating between east and west in the environment does not necessarily require telling right from left. East and west can be discriminated with cues external to the individual (e.g., by noting the direction in which the sun rises and sets). As children, we are typically taught to associate east with right and west with left (Lord, 1941), an association that is reinforced by the common orientation of topographic maps with east to the right and west to the left.

If we are interpreting the demands of the go/no-go task correctly, the results of Experiment 6 suggest that accessing the words "right" and "left" may elicit the right-left effect automatically. This, in turn, suggests that the semantic representation of the words "up" and "down" may be simpler or more comprehensible than the representation for "right" and "left." Based on Experiment 5, evidence for the words "north" and "south" having simpler representation than "east" and "west" is equivocal.

There are two possible explanations for why the words "up" and "down" might be comprehended faster than "right" and "left." First, as suggested by Maki et al. (1979), the labels "right" and "left" may be attached to ambiguous and ill-defined concepts. Spatial labels function to describe location with respect to a particular point of reference. If the point of reference has well-defined right-left coordinates, location with respect to the point of reference is ill-defined when the labels "right" and "left" are used. For example, the statement "The ball is to the right of John" describes the ball's location with respect to John. However, it is unclear whether "to the right of" means on John's right or to the speaker's right. The ball's location is particularly troublesome if John happens to be facing the speaker. Maki et al. propose that such equivocalness is particularly disruptive when the child is first learning to apply the labels "left" and "right," and it may have lasting residual effects on how the words are represented in semantic memory.

Second, the idea of a simpler representation for up and down in reminiscent of H. Clark's (1973) principle of lexical marking, in which " + " and "-" polarity markers are assigned to words within spatial dimensions to denote extent or lack thereof. Empirical data have substantiated the psychological validity of the binary polarity marker (Chase \& H. Clark, 1971; H. Clark, 1969) by demonstrating that unmarked terms (i.e., those assigned +polarity) are processed faster than marked terms (i.e., those assigned -polarity). Given such precedent, it is logical to induce from the extant human performance data that the latency advantage of up and down over right and left reveals the operation of a binary marker representing spatial dimensionality. It has been common within the linguistic literature (Bierwisch, 1967; Leech, 1970) to classify spatial dimensions with a binary vertical marker such that (+vert) corresponds to the vertical dimension and (-vert) to the horizontal. However, in keeping with the universal primitives hypothesis (e.g., see E. Clark, 1973), we suggest that a biologically more significant classification might reflect the anatomy of the human organism, specifically, the bilateral symmetry of the human organism from right to left and the structural symmetry from head to toe and front to back. In accordance with Corballis and Beale's (1976) theory of bilateral symmetry, the young child who has difficulty recognizing which of a pair of mirror image stimuli is which would have trouble attaching the labels "right" and "left" to the mirror image sides of the body. The mirror image equivalence experienced by the child may be the equivalent of or somehow isomorphic with a (-asym) marker denoting the symmetrical right-to-left axis of the human body. In contrast, the front-to-back and head-to-toe axes of the body are coincident with both perceptual (e.g., H. Clark, 1973) and motor asymmetries. The cognitive salience of these asymmetries may be mirrored in a (tasym) marker representing the front-back and up-down egocentric spatial dimensions.

It may be that the sets of spatial labels we have used in these experiments lie on a continuum with respect to egocentricity. In general, the more egocentric the labels are, the more likely they are to elicit a right-left effect. The clock numerals would be the least egocentric. They are rarely mapped onto the body coordinates when wayfinding or describing direction (an exception might be small-craft boat and plane navigation). As a consequence, the clock numerals are normally thought of as describing absolute location on the fact of the clock and are rarely thought of as associated with the right and left sides of the body. Hence, even when the task requires the direction of attention to spatial location (Experiment 3 ), response to the clock numerals is apparently not mediated by an internal sense of right and left. Intermediate on the continuum are the compass labels. Although the compass labels describe an absolute frame of spatial reference, they are commonly mapped onto the body axes when orienting in the environment. When responding to these labels, the subject apparently references an internal sense of right and left when the task requires egocentric orienting responses. Most egocentric, of course, are the labels "up," "down," "left," and "right." Use of these labels may be automatically mediated by an internal sense of relative position.

Alternatively, the differential effects of the spatial labels may have a developmental explanation. Since learning the egocentric labels is ontogenetically prior to 
learning the clock-face and compass labels, the latter terms may be mastered at a time when the child is experiencing less difficulty with telling right from left. As a consequence, the clock-face and compass terms may be less ambiguous and therefore less likely to elicit a right-left effect than are the egocentric labels.

\section{GENERAL DISCUSSION}

The present experiments demonstrate that right-left confusion arises when adults use verbal labels to discriminate orientation or relative location and that the effect is contingent upon the task and the set of labels used. That is, the difficulty seems to lie in using directional labels such as "east" and "west" or "right" and "left." We found no support for the thesis that the locus of the right-left effect is in the perceptual encoding stage of information processing. These results are thus in accordance with Corballis and Beale's (1976) thesis that telling right from left is not a perceptual but a recognition problem. However, the difficulty adults appear to have with telling right from left is not coincident with the difficulty predicted given Corballis and Beale's theoretical criteria of telling right from left. According to the theoretical position espoused by Corballis and Beale, a perfectly bilaterally symmetric organism would be unable to encode or decode right-left information. Hence it is the encoding and decoding of right-left differences that characterizes the organism's ability to tell right from left. The labels used in the task are merely incidental. In the present study, we have disentangled the labeling process from the process of encoding and decoding right-left differences, and we have consistently found the former rather than the latter to be the determining factor in eliciting right-left confusion.

Consider, for example, Experiments 4-6, in which a mirror image discrimination task was used to assess rightleft confusion in the adult. According to bilateral symmetry theory, perceptual events lose their right-left orientation when encoded in memory. As a consequence, mirror images are represented in long-term memory as equivalent, and recognition of the left-right orientation of a stimulus is impaired. There is nothing in the theory to suggest that there should be any effect due to the particular labels used when discriminating mirror image stimuli; that is, right-left confusion should occur regardless of the labels employed. The results of Experiments 4-6 do not support this prediction. When adults discriminate between right-left mirror images, the label used determines whether the effect occurs. That is, there was no right-left effect when the label was the word "go," but there was such an effect when the label was one of the directional words "up," "down," "left," and "right." (The data when the labels were the compass directions "north," "south," "east," and "west" were not clear cut.) These results suggest that recognition of a mirror image stimulus per se is not impaired in the adult. However, there is a corpus of literature suggesting that right-left mirror images are recognized as equivalent by children. Hence orientation information may be represented in the memory system of the adult in a way that is qualitatively different from that of the child.

The results of Experiments 1-3 also show a certain incompleteness in bilateral symmetry theory. The theory predicts that performance should be impaired when bilaterally symmetric organisms make a left response to one stimulus or label and a right response to another. Once again, there is nothing in the theory to suggest that the labels themselves should matter. In the responsedifferentiation task used in Experiments 1-3, a verbal label signaled the subject where to direct his attention in the display in order to respond correctly. When the labels referred to direction on the horizontal axis, subjects responded by directing attention either to the right or to the left. Bilateral symmetry theory would appear to lead to the prediction that the movement of attention to the right or left should be the determining factor in eliciting the effect. However, the determining factor in our experiments was the verbal label, not the implicit left-right response. It could be argued that when map axes were rotated 90 or $270 \mathrm{deg}$, subjects mentally rotated the axes back to 0 deg and then directed the mind's eye to the right or left of the upright image. However, such an argument is vitiated by both the results from the 180-deg rotation condition and the results of Experiment 3. In Experiment 3, subjects showed no right-left confusion when the labels 3 and 9 directed attention to the right and left, respectively.

In summary, when the effect of processing right-left differences is disentangled from the effect of verbal labels, it is the directional label and not the right-left difference per se that adults have difficulty with. These results are inconsistent with Corballis and Beale's (1976) postulate that the labels are arbitrary and bilateral symmetry is fundamental to right-left confusion. However, the theory could handle the discrepancies in part by assuming that adults have developed into functionally asymmetric organisms. For example, the facility with which adults recognize right-left mirror images could be explained by the development of a mechanism that either inhibits homotopic interhemispheric transfer or attenuates the mirror image reversed code. However, as it stands, the theory cannot easily account for the finding that the nature of the verbal label used to describe location or direction is a determining factor in eliciting right-left confusion.

\section{REFERENCES}

Bierwisch, M. Some semantic universals of German adjectives. Foundations of Language, 1967, 3, 1-36.

Chase, W. G., \& Clark, H. H. Semantics in the perception of verticality. British Journal of Psychology, 1971, 62, 311-326.

Clark, E. What's in a word? On the child's acquisition of semantics in his first language. In T. E. Moore (Ed.), Cognitive development and the acquisition of language. New York: Academic Press, 1973.

Clark, H. H. Linguistic process in deductive reasoning. Psychological Review, 1969, 76, 387-404. 
Clark, H. H. Space, time, semantics, and the child. In T. E. Moore (Ed.), Cognitive development and the acquisition of language. New York: Academic Press, 1973.

Clark, H. H., \& Chase, W. G. On the process of comparing sentences against pictures. Cognitive Psychology, 1972, 3, $472-517$

Cooper, L. A., \& Shepard, R. N. Mental rotation of letters. In W. G. Chase (Ed.), Visual information processing. New York: Academic Press, 1973.

Corballis, M. C., \& Beale, I. L. The psychology of left and right. Hillsdale, N.J: Erlbaum, 1976.

Farrell, W. S. Coding left and right. Journal of Experimental Psychology: Human Perception and Performance, 1979, 5, 42-51.

LEECH, G. N. Towards a semantic description of English. Bloomington: Indiana University Press, 1970.

LorTus, G. R. Comprehending compass directions. Memory \& Cognition, 1978, 6, 416-422.

LoRD, F. E. A study of spatial orientation of children. Journal of Educational Research, 1941, 41, 481-505.

MACH, E. [The analysis of sensations] (C. M. Williams, trans.). New York: Dover, 1959. (Originally published, 1886.)

MAKI, R. H. Processing relative locations in a natural space. Bulletin of the Psychonomic Society, 1979, 14, 25-28. (a)

MAKI, R. H. Right-left and up-down are equally discriminable in the absence of directional words. Bulletin of the Psychonomic Society, 1979, 14, 181-184. (b)

Maki, R. H., Grandy, C. A., \& Hauge, G. Why is telling right from left more difficult than telling above from below. Journal of Experimental Psychology: Human Perception and Performance, 1979, 5, 52-67.

Maki, R. H., Maki, W. S., \& Marsh, L. G. Processing locational and orientational information. Memory \& Cognition, 1977, 5, 602-612.

Mille R, J., \& Gheeno, J. G. Goodness-of-fit tests for models of latency and choice. Journal of Mathematical Psychology, 1978, 17, 1-13.

Noble, J. Paradoxical interocular transfer of mirror-image discrimination in the optic chiasm sectioned monkey. Brain Research, 1968, 10, 127-151.
Posner, M. I. Chronometric explorations of mind. Hillsdale, N.J: Erlbaum, 1978.

Shold, M. J., \& EGETH, M. E. Interpreting directions from graphic displays: Spatial frames of reference. In P. A. Kolers, M. E. Wrolstad, \& N. Bouma (Eds.), Processing of visible language (Vol. 2). New York: Plenum, in press.

Shulman, G. L., Remington, R. W., \& McLean, J. P. Moving attention through visual space. Journal of Experimental Psychology: Human Perception and Performance, 1979, 5, 522-527.

STERNBERG, S. The discovery of processing stages: Extensions of Donders' method. Acta Psychologia, 1969, 30, 276-315.

\section{NOTES}

1. Of course, one does not normally seek support for the additivity of main effects from a statistical interaction. However, our situation is unusual in that the logic of the experimental design requires a statistical analysis of the factors rotation condition and verbal label, whereas the experimental hypotheses address the effects of spatial axis and verbal label. That additive main effects of verbal label and spatial axis are equivalent to an interaction between verbal label and rotation condition can be seen by transforming a plot of the additive main effects with spatial axis on the abscissa to a figure containing the same data points with rotation condition on the abscissa.

2. In actuality, this experiment was run before Experiment 5 . At that time, we considered the collection of VOT means to be perfunctory, especially given Farrell's (1979, Experiment 2) finding of no significant differences in VOT for the words "up," "down," "right," and "left." Hence, in Experiment 6, we collected 10 trials per VOT condition and found that our results confirmed Farrell's. When it became evident in piloting Experiment 5 that VOT might be confounding RT, we increased the number of trials per VOT condition to 20 to increase the reliability of the measure.

(Received for publication August 11, 1980; revision accepted January 12, 1981.) 